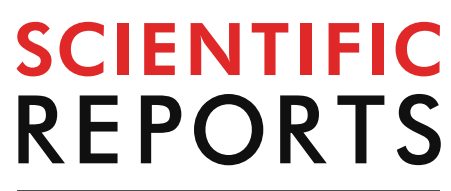

natureresearch

Check for updates

\title{
The complete mitochondrial genome of medicinal fungus Taiwanofungus camphoratus reveals gene rearrangements and intron dynamics of Polyporales
}

Xu Wang ${ }^{1}$, Lihua Jia ${ }^{1}$, Mingdao Wang ${ }^{1}$, Hao Yang ${ }^{1}$, Mingyue Chen ${ }^{1}$, Xiao Li $^{1}$, Hanyu Liu ${ }^{1}$, Qiang $\mathrm{Li}^{2,3 凶} \& \mathrm{Na} \mathrm{Liu}^{1,3 凶}$

Taiwanofungus camphoratus is a highly valued medicinal mushroom that is endemic to Taiwan, China. In the present study, the mitogenome of $T$. camphoratus was assembled and compared with other published Polyporales mitogenomes. The T. camphoratus mitogenome was composed of circular DNA molecules, with a total size of $114,922 \mathrm{bp}$. Genome collinearity analysis revealed large-scale gene rearrangements between the mitogenomes of Polyporales, and $T$. camphoratus contained a unique gene order. The number and classes of introns were highly variable in 12 Polyporales species we examined, which proved that numerous intron loss or gain events occurred in the evolution of Polyporales. The Ka/Ks values for most core protein coding genes in Polyporales species were less than 1 , indicating that these genes were subject to purifying selection. However, the rps3 gene was found under positive or relaxed selection between some Polyporales species. Phylogenetic analysis based on the combined mitochondrial gene set obtained a well-supported topology, and T. camphoratus was identified as a sister species to Laetiporus sulphureus. This study served as the first report on the mitogenome in the Taiwanofungus genus, which will provide a basis for understanding the phylogeny and evolution of this important fungus.

Taiwanofungus camphoratus, belonging to Polyporales, Basidiomycota, is an endemic mushroom of Taiwan, China, which is restricted to the endemic aromatic tree Cinnamomum kanehirai ${ }^{1}$. In the last few decades, $T$. camphoratus was included in folk medicine in Asia and has shown remarkable effectiveness in the treatment of inflammatory disorders, cancer, hypertension and hepatitis ${ }^{2-6}$. The total market value of T. camphoratus products has grown rapidly, with market value reported to exceed $\$ 100$ million annually in $2014^{7}$. Many active secondary metabolites were isolated from the mycelium or fruiting bodies of T. camphoratus, such as triterpenoids, lipids, and benzenoids, showing excellent anti-inflammatory, hepatoprotective, antioxidant, radioprotective, and chemoprevention activities $^{8-11}$. T. camphoratus shares many common characters with Antrodia and Antrodiella, which caused its taxonomic confusion in the past ${ }^{1}$. Phylogenetic analysis based on LSU rDNA sequence indicated that $T$. camphoratus should be proposed as a new genus of lignicolous polypore ${ }^{1}$. The genome of T. camphoratus has been published to reveal the sexual development and metabolic synthesis of T. camphoratus. However, the characterization of the mitogenome of Taiwanofungus camphoratus has not been conducted to date.

Mitochondria generate most of the cell's supply of adenosine triphosphate (ATP) in eukaryotes ${ }^{12}$. Most eukaryotes have their own mitochondrial genomes (mitogenomes), which are believed to be derived through endosymbiosis from the ancestral alpha-proteobacterium ${ }^{13,14}$. As the "second genome" of eukaryotes, mitogenomes have many characteristics different from nuclear genomes. For example, each eukaryotic cell contains a large number of mitochondrial organelles, and each mitochondrial organelle has multiple mitochondrial genomes ${ }^{15}$.

${ }^{1}$ College of Life Sciences, Henan Agricultural University, Zhengzhou 450002, Henan, China. ${ }^{2}$ School of Food and Biological Engineering, Chengdu University, Chengdu 610106, Sichuan, China. ${ }^{3}$ Present address: College of Life Sciences, Henan Agricultural University, Zhengzhou 450002, Henan, China. ${ }^{\boxplus e m a i l}$ leeq110@126.com; naliu@ henau.edu.cn 
In addition, its rapid evolutionary rate, and multiple available molecular markers have made the mitochondrial genome a powerful tool for studying phylogeny and population genetics ${ }^{16,17}$. With the rapid development of next-generation sequencing technology, more and more mitochondrial genomes have been obtained ${ }^{18-22}$. So far, more than 10,000 complete mitochondrial genomes have been released in the NCBI reference database, including animal, plant and fungal mitogenomes. These available mitogenomes promote the phylogeny and taxonomy of eukaryotes ${ }^{23-25}$. However, compared with its animal counterparts, the mitochondrial genome of fungi has been less studied ${ }^{26}$. As an important group of eukaryotes, fungus is widely distributed in the world. It is estimated that more than 2.2 million of fungal species exist in nature ${ }^{27}$, which play an important role in maintaining the carbon and nitrogen cycle in nature, maintaining the healthy development of forest ecosystems, and providing food sources for human beings ${ }^{7,28,29}$. However, fewer than 800 fungal mitogenomes are available, with available mitogenomes of Basidiomycetes are even less $(<130)$. The few available mitogenomes limit our overall understanding of evolution, phylogeny, and taxonomy of the fungal lineages, because phylogenies based on mitochondrial genes and nuclear genes are not always consistent ${ }^{30}$.

Up to now, only 11 complete mitogenomes of Polyporales species have been published, including seven species in Ganoderma genus ${ }^{31-34}$, one in Trametes ${ }^{35}$, one in Phlebia ${ }^{36}$, one in Laetiporus ${ }^{37}$, and one in Fomitopsis ${ }^{38}$. The sizes of these mitogenomes varied greatly, ranging from 60,635 to $156,348 \mathrm{bp}$. This is thought to be due to variations of intron numbers. Introns in the mitogenome of fungi can be divided into two groups, namely the group I and the group II. Group II intron excision occurs in the absence of GTP and involves the formation of a lariat, which were different from group I intron. These introns were found distributed in the cox, cob, nad and rRNA genes of the fungal mitogenomes ${ }^{39-42}$, which could modify the organization and size of mitogenome ${ }^{39,43,44}$. In addition, a variety of homing endonuclease genes have been found in fungal introns, such as the LAGLIDADG endonuclease and GIY endonucleases ${ }^{45,46}$, the products of which have homing endonuclease activities. However, the origin, evolution, and dynamics of the mitochondrial introns in the order Polyporales are still unknown.

The mitogenomes of fungi are significantly different from that of other eukaryotes. The genome size, gene arrangement, repetitive sequences, introns, intergenic regions, and open reading frames (ORFs) of fungal mitogenomes varied greatly between species and even between intraspecific species ${ }^{18,47,48}$. Despite the large variations, most Basidiomycete mitogenomes were found containing 14 core protein coding genes (atp6, atp 8, atp $9, \operatorname{cox} 1$, $\operatorname{cox} 2$, cox3, cob, nad1, nad2, nad3, nad4, nad4L, nad5, and nad6) for energy metabolism, one rps3 gene for transcriptional regulation $^{49}, 22-36$ tRNA genes, and two rRNA genes ${ }^{50,51}$. Repeated sequences in the mitogenome, gene arrangement, and structures of tRNAs can provide important information to reveal the evolutionary and phylogenetic relationships of fungi ${ }^{39,52,53}$.

In the present study, the mitogenome of T. camphoratus were assembled and annotated. The aims of this study are (1) to reveal variations and conservation of gene content, genome organization, and gene order between $T$. camphoratus mitogenome and other Polyporales species; (2) to provide insights into the evolution and dynamic changes of introns in Polyporales mitogenomes; (3) to reveal the phylogenetic status of T. camphoratus among various Agaricomycetes species based on the combined mitochondrial gene set. The mitogenome of T. camphoratus will allow further study of the taxonomy, phylogenetics, and evolutionary studies of this important medical fungus and other closely related species.

\section{Results}

Genome features and protein coding genes. The complete mitogenome of T. camphoratus was composed of circular DNA molecules, with the total size of 114,922 bp (Fig. 1). GC content of the T. camphoratus mitogenome was $26.01 \%$. The AT skew was negative in the mitogenome of T. camphoratus, where the GC skew was positive (Table S1). Fifteen core protein coding genes were detected in the T. camphoratus mitogenome, including atp6, atp8, atp9, cob, cox1, cox2, cox3, nad1, nad2, nad3, nad4, nad4L, nad5, and nad6 for energy metabolism, and one rps3 gene for transcriptional regulation (Table S2). In addition, the T. camphpratus mitogenome contained 19 non-intronic ORFs, which included an RNA polymerase gene, two DNA polymerase genes and 16 PCGs with unknown function. Twenty-four introns were detected in the mitogenome of T. camphoratus, which were distributed in the host gene of coxl, cob, nad1, and $r n l$. All of these introns belong to the group I. Twenty-three intronic ORFs were found located in these introns, which encoded LAGLIDADG and GIY-YIG homing endonucleases.

RNA genes in the $T$. camphoratus mitogenome. Two rRNA genes were detected in the T. camphoratus mitogenome, namely the large subunit ribosomal RNA ( $r n l)$, and the small subunit ribosomal RNA ( $r n s$ ) (Table S2). The mitogenome of T. camphoratus contained 27 tRNA genes, which were folded into classical cloverleaf structures (Fig. 2). The mitogenome of T. camphoratus contained 2 tRNAs with different anticodons coding for serine, arginine, leucine and 3 tRNAs with different anticodons coding for glutamate. In addition, the T. camphoratus mitogenome contained 3 tRNAs with the same anticodon that coded for methionine. The length of individual tRNAs ranged from 71 to $86 \mathrm{bp}$, which was mainly due to the variation of extra arm. A ribonuclease P RNA $(r n p B)$ gene was found in the T. camphoratus mitogenome, with the length of $329 \mathrm{bp}$.

Overlapping nucleotides and mitogenome composition. Only one overlapping nucleotide was detected in the mitogenome of T. camphoratus, which was located across the neighboring genes nad $4 L$ and nad5 (1 bp) (Table S2). We detected $41,887 \mathrm{bp}$ intergenic sequences in the mitogenomes of T. camphoratus. The length of these intergenic sequences ranged from 0 to $2903 \mathrm{bp}$, and the longest intergenic sequence was located between the trnW and orf 292 gene. Intergenic regions accounted for the largest proportion of the T. camphoratus mitogenome, reaching 36.45\% (Fig. 3), which showed that the mitogenome of T. camphoratus had a relatively relaxed structure. The intronic region occupied the second largest proportion of the T. camphoratus 


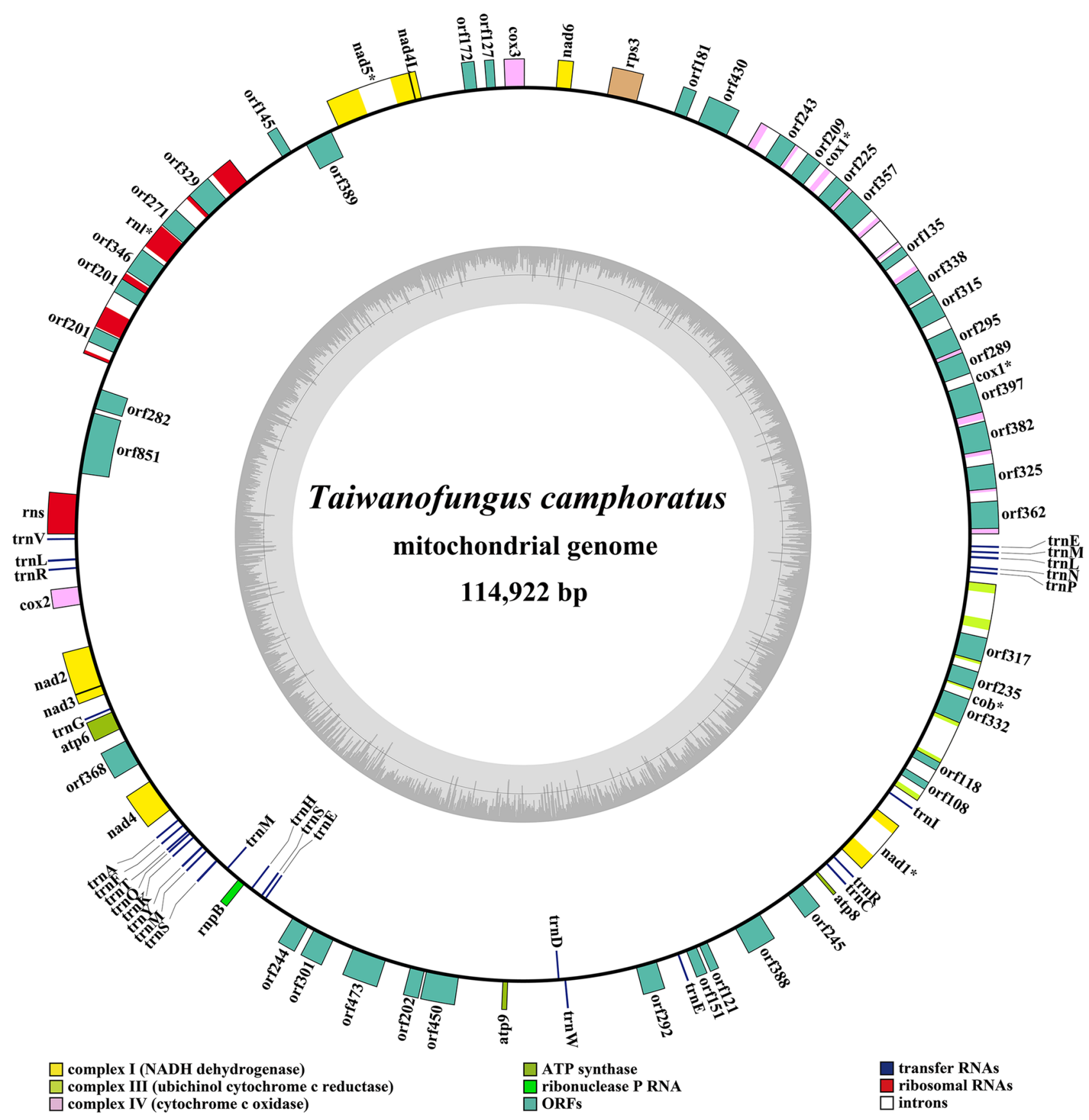

Figure 1. Circular map of the mitochondrial genome of Taiwanofungus camphoratus. Genes are represented by different colored blocks. Colored blocks outside each ring indicate that the genes are on the direct strand, while colored blocks within the ring indicates that the genes are located on the reverse strand.

mitogenome, accounting for $29.94 \%$. The protein coding region accounted for $27.56 \%$ of the T. camphoratus mitogenome. The RNA region ( $\mathrm{rRNA}+\mathrm{tRNA}+r n p B)$ accounted for $6.05 \%$ of the entire mitogenome.

Codon usage analysis. ATG was the most commonly used start codon for the 15 core PCGs in the 12 Polyporales species we examined (Table S3). Eleven of the 15 core PCGs used ATG as start codons in all the 12 Polyporales mitogenomes we detected. GTG was the most commonly used start codon in cob gene of the 12 Polyporales mitogenomes. In addition, ATA and TTG were also used as start codons in cox2, nad1 and nad6 gene of some Polyporales species. TAA was most commonly used as stop codons of core PCGs in 12 Polyporales species, followed by TTG and AGT. We found that all core PCGs in T. camphoratus used ATG as start codons and TAA as stop codons.

Codon usage analysis indicated that the most frequently used codons in the T. camphoratus mitogenome were TTT (for phenylalanine; Phe), AAA (for lycine; Lys), TTA (for leucine; Leu), AAT (for asparagine; Asn), 

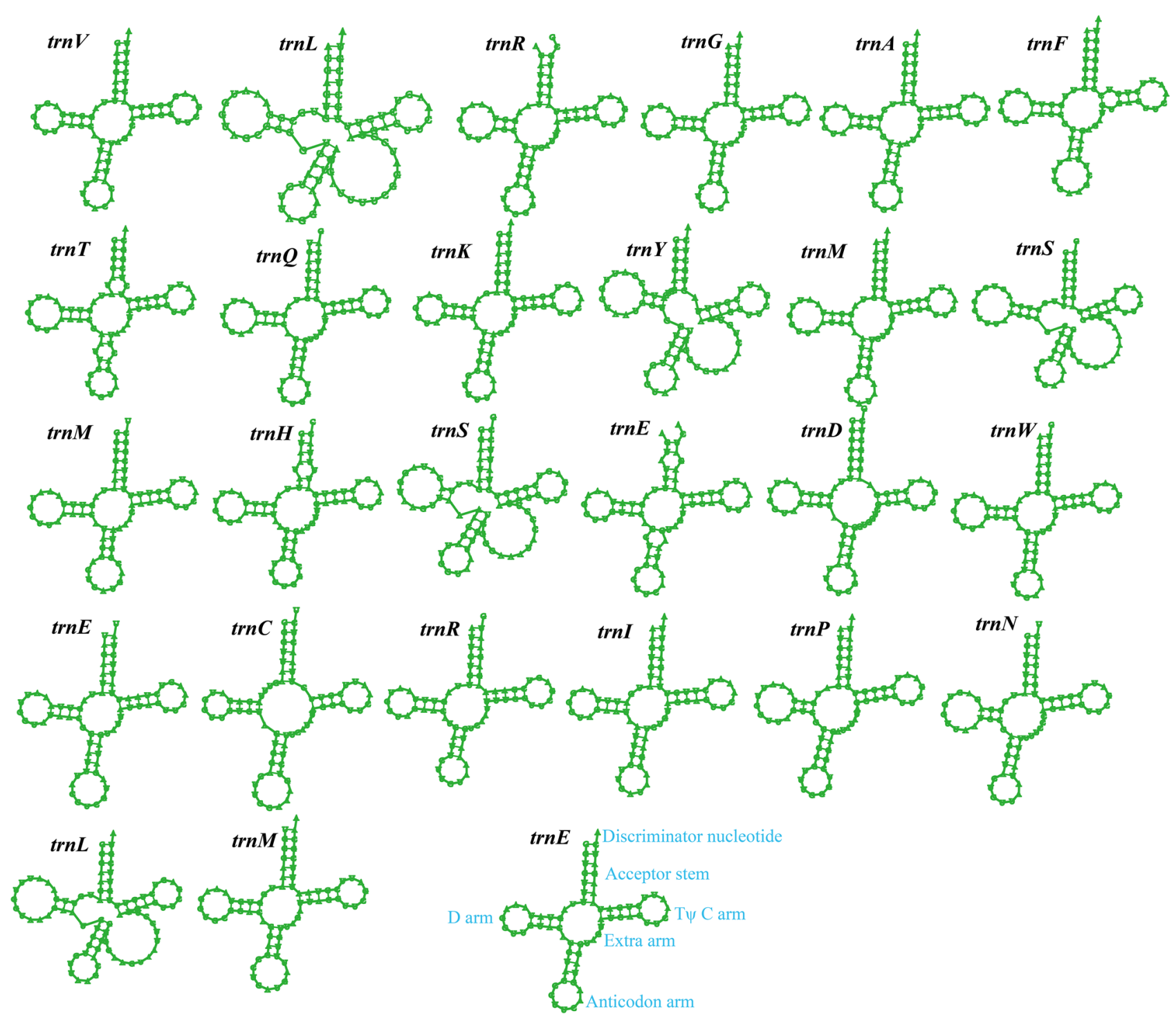

Figure 2. Putative secondary structures of the 27 tRNA genes identified in the mitochondrial genome of Taiwanofungus camphoratus. All genes are shown in order of occurrence in the mitochondrial genome of Taiwanofungus camphoratus, starting from trnV. tRNA structures were determined using MITOS.

ATT (for isoleucine; Ile), and TAT (for tyrosine; Tyr) (Fig. 4 and Table S4). The frequent use of A and T in codon contributed to the high AT content in the T. camphoratus mitogenome (average: $73.99 \%$ ).

Repetitive sequences in the mitogenome. Comparing the whole mitogenome of T. camphoratus with itself via BLASTn search, we identified 45 repeat sequences in the mitogenome of T. camphoratus (Table S5). The length of these repeat sequences ranged from 28 to $902 \mathrm{bp}$, with pair-wise nucleotide similarities ranging from 74.72 to $100 \%$. The largest repeats were observed in the third introns and fourth introns of the $r n l$ gene. The second largest repeats were found located in the intergenic region between orf145 and $\mathrm{rnl}$, as well as in intergenic region between orf388 and orf245, with each repeating sequence 840 bp long. Repeat sequence accounted for $9.44 \%$ of the T. camphoratus mitogenome.

Using the Tandem Repeats Finder, we identified 55 tandem repeats in the T. camphoratus mitogenome (Table S6). The longest tandem repeat sequence was observed in the intergenic region between $\operatorname{nad} 4$ and $\operatorname{trn} A$, comprising $79 \mathrm{bp}$. Most of the tandem repeat sequences were duplicated once or twice in the T. camphoratus mitogenome, with the highest replication number 15 . Tandem repeat sequences accounted for $2.04 \%$ of the $T$. camphoratus mitogenome. REPuter identified 27 forward, 7 palindromic, and 16 reverse repeats in the mitogenome of T. camphoratus, accounting for $6.33 \%$ of the entire mitogenome (Table S7).

Comparative genome analysis and gene rearrangement. The mitogenome of $T$. camphoratus was the fourth largest among the published mitogenomes in Polyporales, which was smaller than Phlebia radiata ${ }^{36}$, G. calidophilum, and G. applanatum, and larger than Trametes cingulata ${ }^{35}$, Fomitopsis palustris ${ }^{38}$, Laetiporus sulphureus $^{37}$ and other Ganoderma spp. species (Table S1). The GC content of the T. camphoratus mitogenome was 


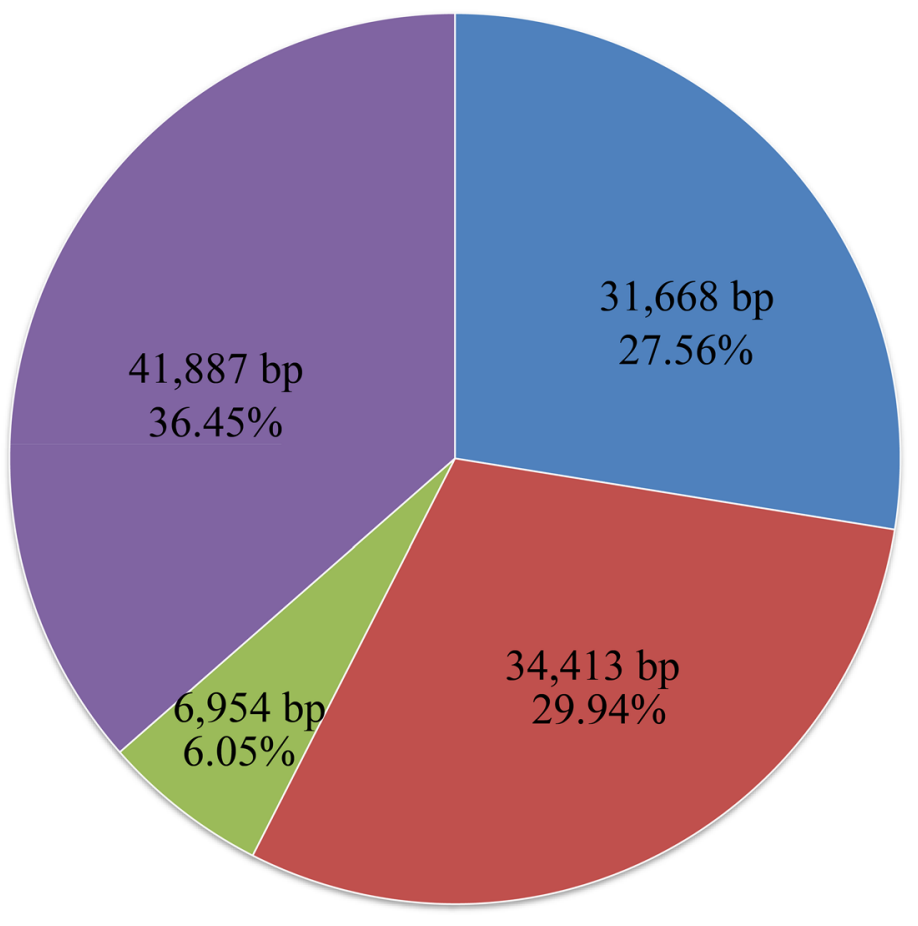

$\square$ Protein coding region $\quad$ Intronic region $\quad$ RNA sequence $\quad$ Intergenic region

Figure 3. The protein-coding, intronic, intergenic, and RNA gene region proportions of the entire mitochondrial genome of Taiwanofungus camphoratus.

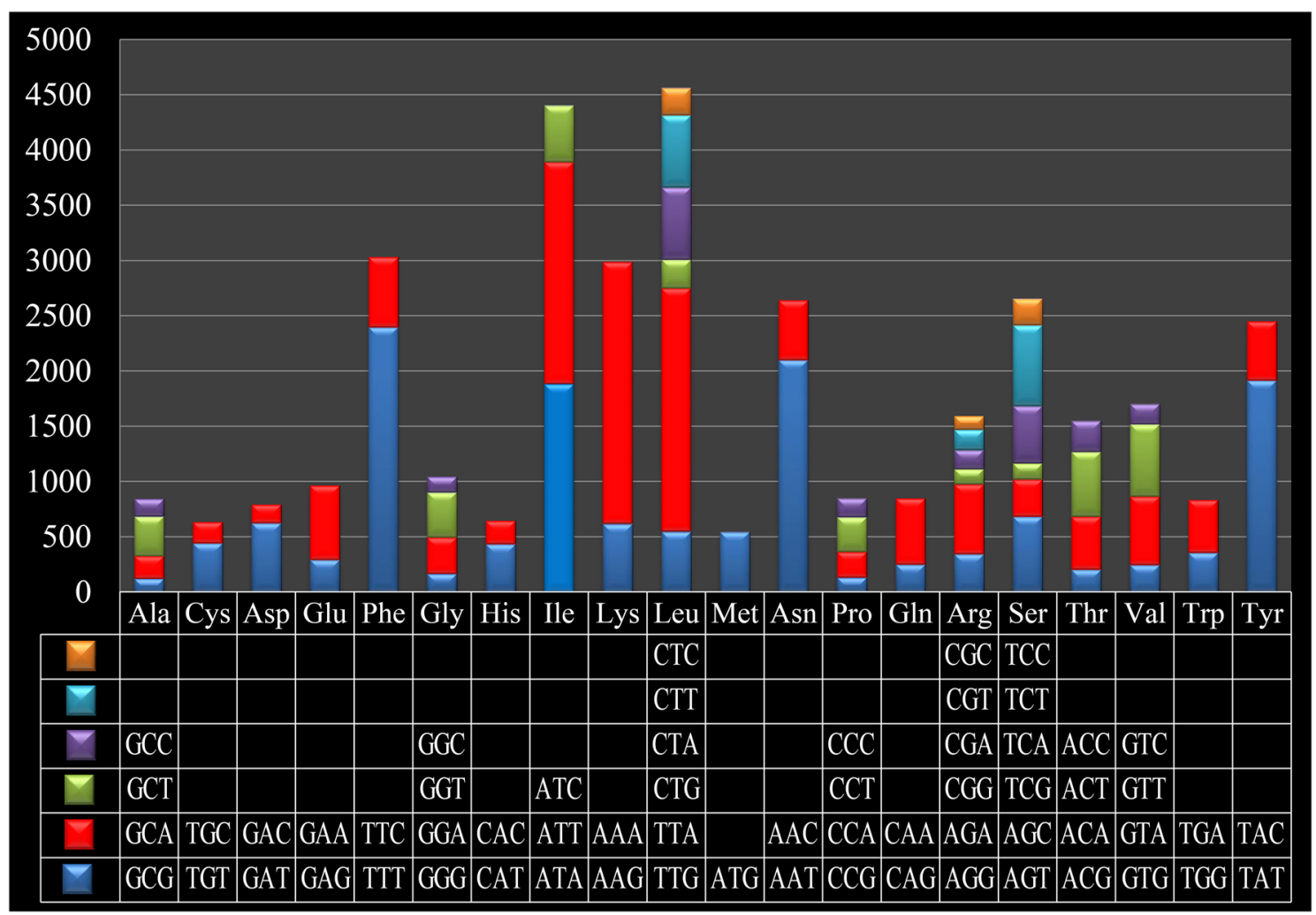

Figure 4. Codon usage in the mitochondrial genome of Taiwanofungus camphoratus. Count of codon usage is plotted on the $y$-axis. 


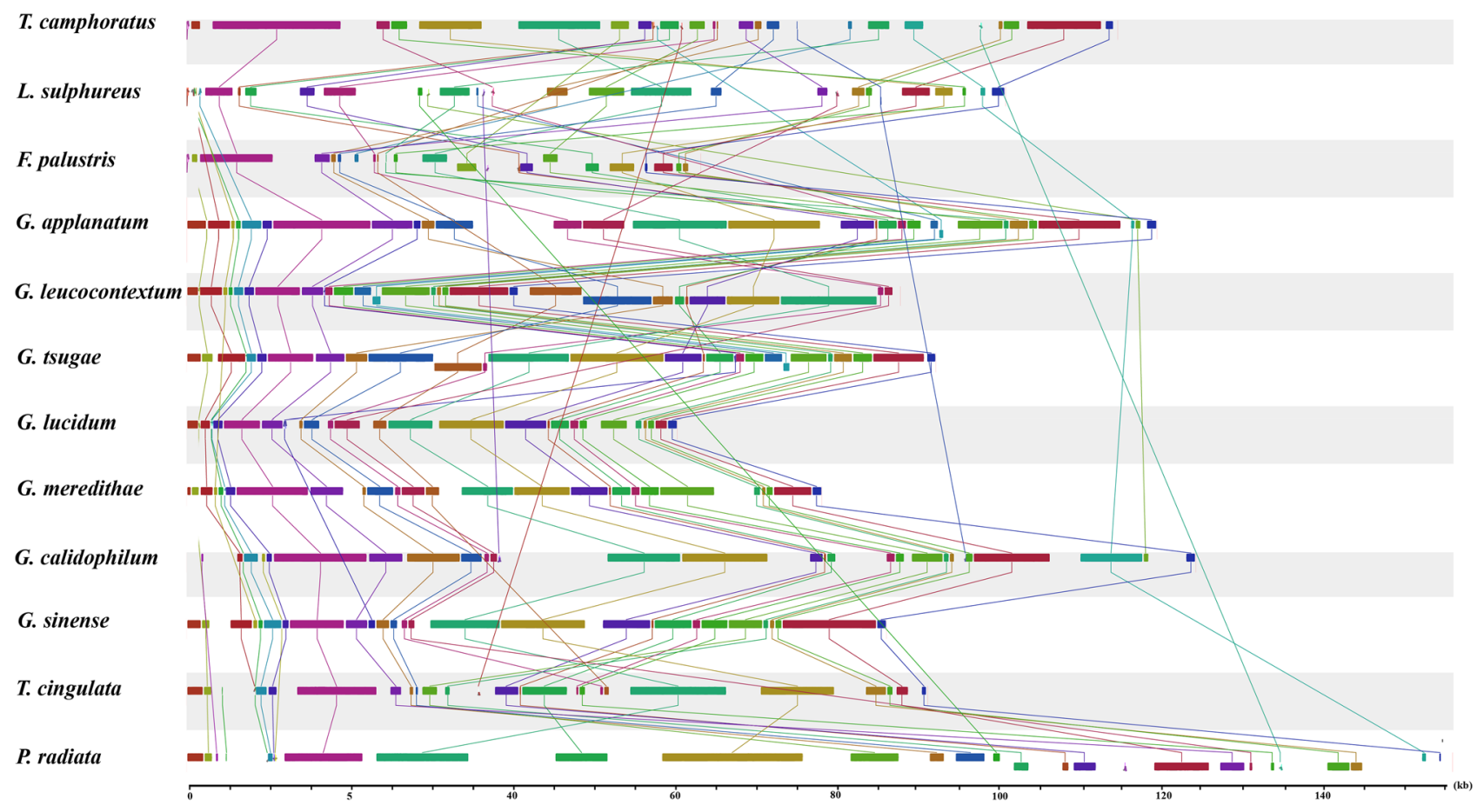

Figure 5. Co-linearity analysis of 12 Polyporales mitogenomes. Homologous regions were connected with straight lines of the same color. The sizes and relative positions of the homologous regions varied among the mitogenomes.

in the middle among all Polyporales mitogenomes. Of the 12 Polyporales we tested, only T. camphoratus and $L$. sulphureus had negative AT skews, while the others were positive. The GC skew of the T. camphoratus mitogenome was positive, just like most of the Polyporales. Thirty-four non-intronic ORFs and 24 introns were found in the T. camphoratus mitogenome, which were smaller than the 79 non-intronic ORFs and 36 introns in the $P$. radiata mitogenome that made $P$. radiata the largest mitochondrial genome in the Polyporales. All 12 Polyporales mitogenomes detected contain 2 rRNA genes. The number of tRNA genes in the mitogenomes of Polyporales ranged from 25 to 29.

Genomic collinearity analysis showed that the mitogenome of T. camphoratus had 28 homologous regions with other Polyporales mitogenomes (Fig. 5). The relative positions of these homologous regions were highly variable between Polyporales species, suggesting that frequent gene rearrangements had occurred during the mitogenome evolution of Polyporales. In addition, we found that the gene arrangement was highly conserved in the genus Ganoderma and highly variable at family or order levels. Gene arrangement analysis involving all core PCGs, rRNA genes and tRNAs indicated that T. camphoratus had a unique gene order, which showed its unique evolutionary characteristics in Polyporales species.

Dynamics of introns in cox 1 and cob genes of Polyporales. The number and size of introns were highly variable in mitogenomes of Polyporales, which promoted the size and organization dynamic changes of Polyporales. A total of 271 introns were detected in 12 published mitogenomes of Polyporales, with individual species containing 6-36 introns. These introns distributed in several host genes, including cox $1, \operatorname{cob}, \operatorname{rnl}, \operatorname{cox} 2$, and nad1. The cox 1 gene contained 130 introns, accounting for $47.97 \%$ of the total number of introns, followed by cob gene, which contained 48 introns, accounting for $17.71 \%$ of the total number of introns. According to previous studies ${ }^{43}$, each intron was considered as a mobile genetic element. Group I introns in fungi could be classified into different position classes (Pcls) according to their insertion site in the coding region of $\operatorname{cox} 1$ or cob gene. The same Pcls were considered to be orthologous, and had high nucleotide similarities. Different Pcls had lower sequence similarities, and with non-homologous homing endonucleases. In the present study, we classified group I introns in cox 1 genes of the 12 Polyporales species into 26 Pcls according to methods described by Férandon et al. ${ }^{43}$. As shown in Fig. 6, the same letters denoted the same Pcls. T. cingulata contained the most Pcls (15) in the coxl gene, followed by G. calidophilum and G. applanatum. G. lucidum contained the least Pcls, suggesting that intron loss had occurred in the evolution of $G$. lucidum. Pcls P was the most common intronic classes in the cox1 genes of Polyporales, which presented in 10 of the 12 Polyporales species. Pcls T and D were also widely distributed in cox 1 genes of Polyporales species, which existed in 9 and 8 Polyporales species, respectively. In addition, some rare introns were found in the cox 1 gene of Polyporales, e.g. Pcl Z, which was observed only in the cox 1 gene of $L$. sulphureus. However, this Pcls has been observed in the mitogenome of Agaricus bisporus from Agaricales ${ }^{43}$, suggesting potential horizontal gene transfer events between the two species. Pcl R was observed only in P. radiata from Polyporales, but was observed in cox 1 gene of Rhizophydium sp. 136 ${ }^{43}$. In addition, Pcl Y was also considered to be a rare intron class in Polyporales, which only existed in T. camphora- 

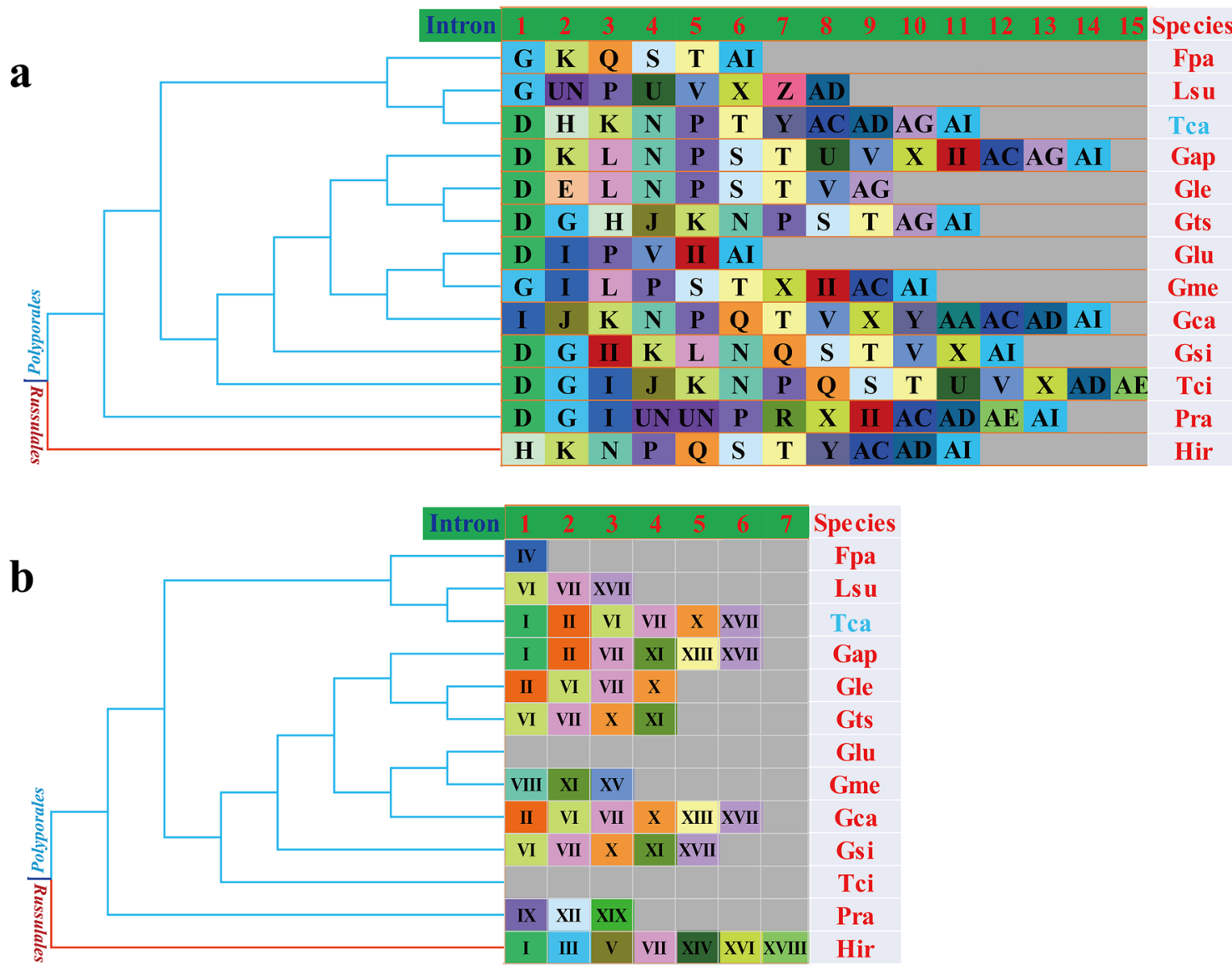

Figure 6. Pcl information of $c o x 1$ gene (a) and cob gene (b) of the 12 Polyporales species. The same Pcl (orthologous intron) is represented by the same letter or the same roman numeral. The phylogenetic positions of 12 Polyporales species were established using the Bayesian inference (BI) method and Maximum Likelihood (ML) method based on 15 concatenated mitochondrial core proteins and 2 rRNA genes. UN indicates that the intron is different from the known introns ${ }^{44}$ in insertion site and sequence similarity. The II in the figure above shows that the intron belongs to the group II intron. Species ID are shown in Supplementary Table S8.

tus and G. calidophilum. The Pcl Y was also observed in distant species Chlorokybus atmophyticus. In addition, three unknown Pcls were found in the mitogenome of Polyporales with no significant nucleotide similarities to reported Pcls.

The introns of cob genes in Polyporales were classified into 18 Pcls according to the insertion sites in the coding region of cob gene (Fig. 6). T. camphoratus, G. applanatum and G. calidophilum contained the most Pcls in cob gene. The cob gene of T. cingulata and G. lucidum was intronless. Pcls VI and VII were considered to be the common Pcls in Polyporales, which existed in at least 6 of the 12 Polyporales species detected. While Pcls IV, VIII, IX, XII, XV, and XIX only existed in one of 12 species, which were considered to be rare introns in the cob gene. However, these rare Pcls showed highly nucleotide similarities with distant species, suggesting frequent gain or loss of cob introns in Polyporales.

Evolution rate of core genes and phylogenetic analyses. Of the 15 core PCGs detected, the rps 3 gene had the highest mean K2P genetic distance among the 12 Polyporales species, followed by nad3 (Fig. 7). The pairwise K2P genetic distance of rps3 and nad3 gene between Polyporales species varied largely. The mean genetic distance of nad $4 \mathrm{~L}$ gene between the 12 Polyporales species was the smallest among the 15 core PCGs detected, indicating that this gene was highly conserved across the mitogenomes. The nad 3 gene had the highest nonsynonymous substitution rate (Ka) of the 15 core PCGs detected, while atp 9 had the lowest rate. The highest synonymous substitutions rate (Ks) was observed in the nad 3 gene, while atp 9 exhibited the lowest Ka value of the 15 PCGs detected. The Ka/Ks values for most core PCGs were less than 1, indicating that these genes were subject to purifying selection. However, the Ka/Ks value of $r p s 3$ gene was observed more than 1 between some species, such as between T. camphoratus and L. sulphureus, between T. camphoratus and F. palustris, as well as between $G$. sinense and G. applanatum, indicating that rps3 gene was under positive selection pressure in some Polyporales species.

Phylogenetic analyses based on the combined mitochondrial gene set ( 15 core PCGs +2 rRNAs), using bayesian inference (BI) and maximum likelihood (ML) methods with the GTR+I+G model of nucleotide substitution, yielded identical and well-supported tree topologies (Fig. 8). All major clades of the trees were found with good 

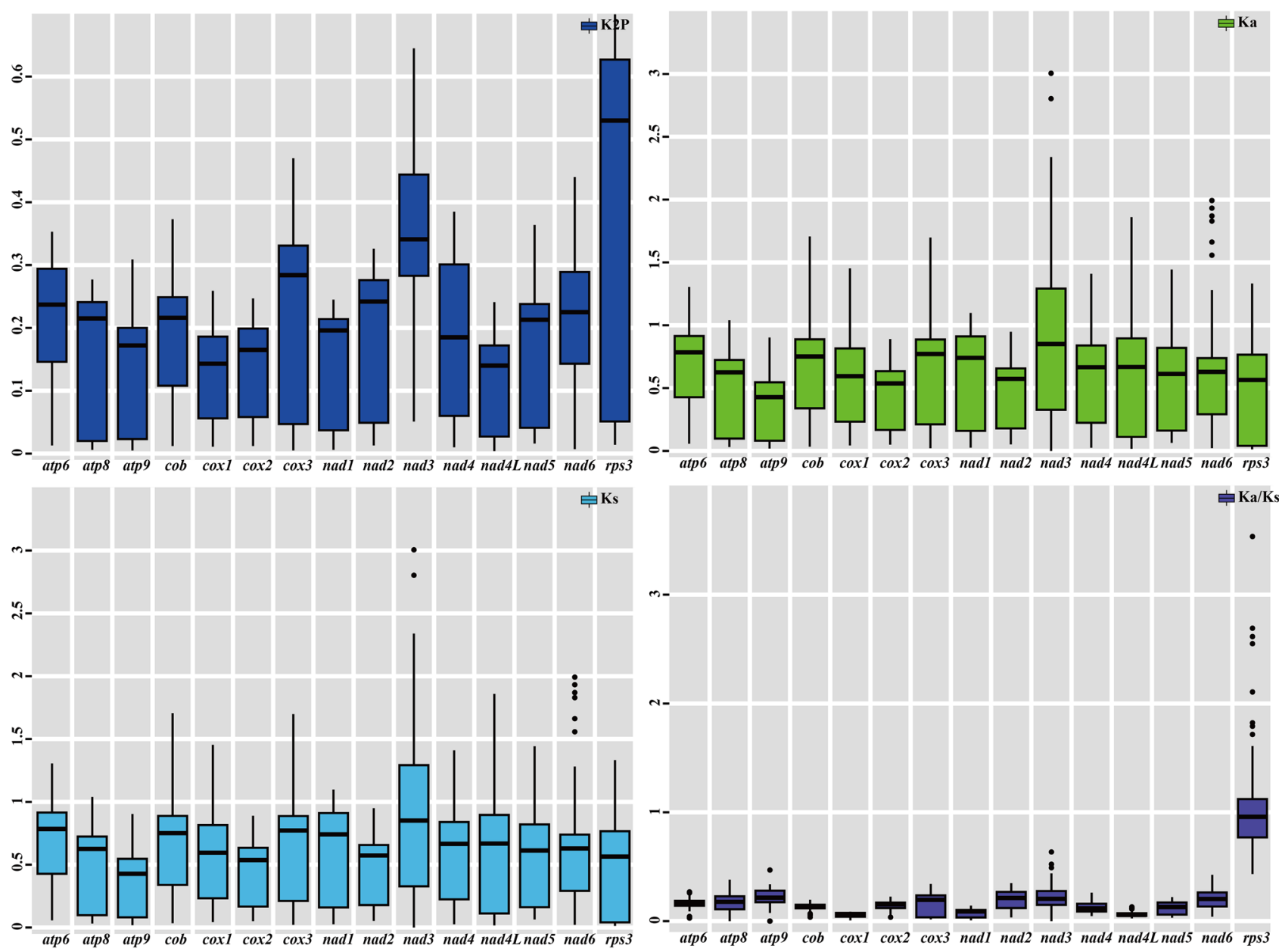

Figure 7. Genetic analysis of 15 protein coding genes conserved in 12 Polyporales mitogenomes. K2P the Kimura-2-parameter distance, $K a$ the mean number of nonsynonymous substitutions per nonsynonymous site, $K s$ the mean number of synonymous substitutions per synonymous site.

support $(\mathrm{BPP}=1.00$ and $\mathrm{BS} \geq 75)$. Based on the phylogenetic analyses, the 25 Agaricomycetes species could be divided into four major clades, corresponding to the orders Polyporales, Agaricales, Russulales, and Cantharellales (Table S8). The 12 Polyporales species could be divided into three groups; one group was composed of one species in the Phlebia genus; the second group was composed of one species in the Trametes genus and seven species in the Ganoderma genus; the third group was recovered as (F. palustris + (T. camphoratus + L. sulphureus)). T. camphoratus was identified as a sister species to L. sulphureus. Phylogenetic studies showed the evolutionary status of the T. camphoratus in the Agaricomycetes class. The results indicated that the combined mitochondrial gene set was suitable as reliable molecular marker for analysis of the phylogenetic relationships among Agaricomycetes species.

\section{Discussion}

As the 'second genome' of eukaryotes, mitochondrial genome plays an important role in aging, death, disease occurrence and stress resistance of eukaryotes ${ }^{54-56}$. Mitochondrial genome organization, core protein coding genes, repetitive sequences, gene arrangement, the number and structure of tRNAs, and open reading frames provided rich genetic information for understanding the genitics and evolution of eukaryotes ${ }^{57-59}$. Mitochondrial genomes have been extensively studied in animals. However, as an important group of eukaryotes, the mitochondrial genomes of fungi have been less studied, especially in Agaricomycetes ${ }^{60}$. As the largest mushroom-forming fungal group, less available Agaricomycetes mitochondrial genomes have limited our understanding of the origin, evolution, and phylogenetic relationships of mushroom-forming fungi. The mitochondrial genome of fungi was thought to have a moderate mutation rate, intermediating to that of plants (highest mutation rate) and animals (lowest mutation rate) ${ }^{61,62}$. It was reported that the mitogenome size, gene arrangement, and repetitive sequences of fungi varied greatly between and within species $39,47,48,63$. The largest known Agaricomycetes mitochondrial genome was Rhizoctonia solani ${ }^{64}$, which was $235.85 \mathrm{~kb}$ long and contained 127 PCGs, 2 rRNA genes and 26 tRNA genes. In Polyporales order, the mitogenome size varied greatly, ranging from 60,635 to 156,348 bp. Accordingly, the non-intronic ORFs ranged from 15 to 79; intron numbers ranged from 6 to 36, and the number of tRNA genes ranged from 25 to 29. Variations in the number and size of these genes contributed to huge variations in the size of the mitochondrial genomes in Polyporales. Previous studies showed that the size variation of mitogenome was 


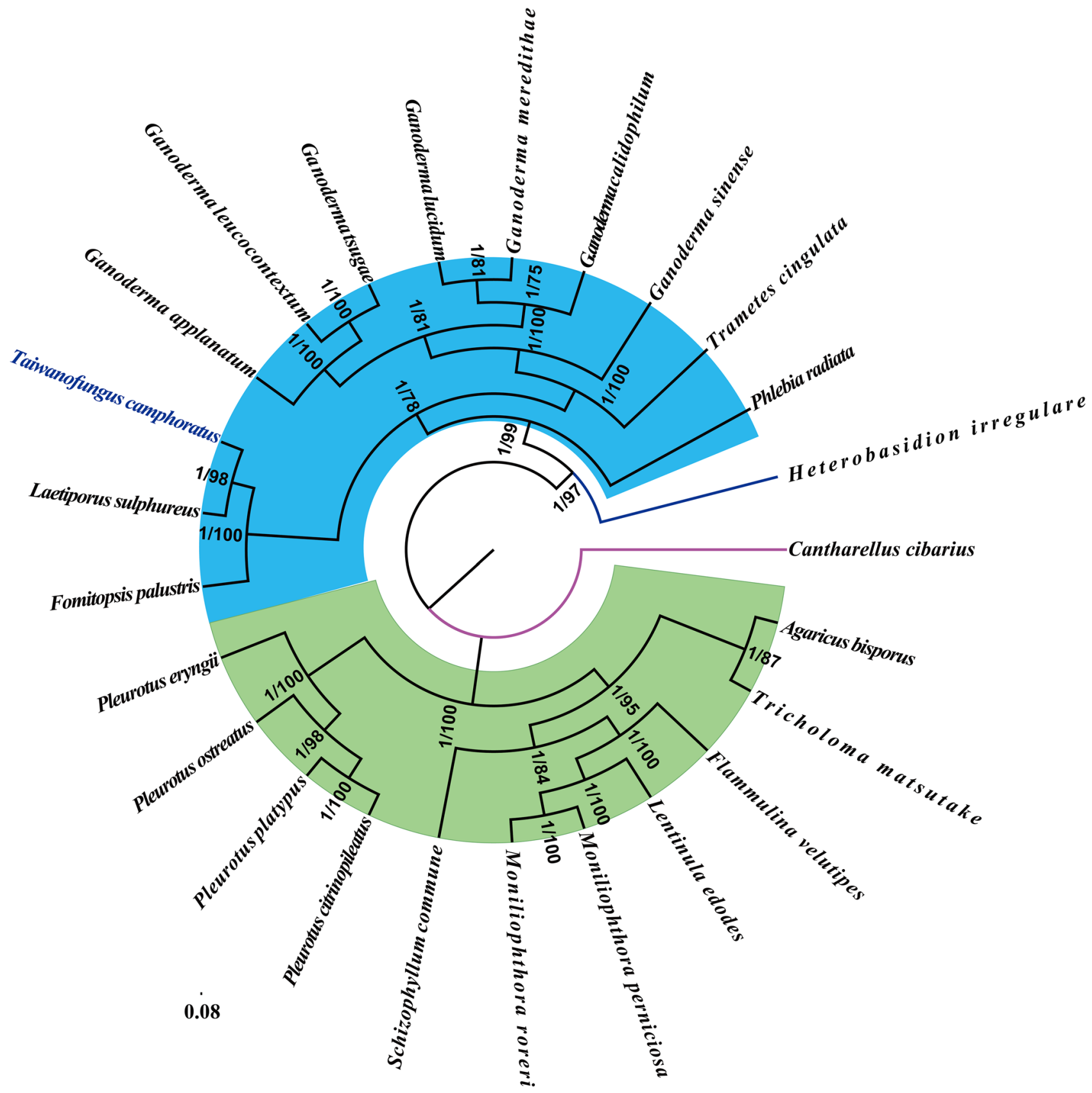

Figure 8. Molecular phylogeny of 25 Agaricomycetes species based on Bayesian inference (BI) and Maximum Likelihood (ML) analyses of 15 protein coding genes and two rRNA genes. Support values are bayesian posterior probabilities (before slash) and bootstrap values (after slash). Species and NCBI accession numbers for genomes used in the phylogenetic analysis are provided in Supplementary Table S8.

closely associated with intron number variation ${ }^{43}, 44$, which was consistent with this study. In the present study, the T. camphoratus genome size, open reading frames, GC content, GC skew and AT skew were not consistent with other Polyporales species, indicating the unique evolutionary characteristics of T. camphoratus mitogenome.

In the present study, we found that most introns in Polyporales belonged to the group I, which was different from plants ${ }^{61}$. A total of 271 introns were found in the 12 Polyporales species, most of which were distributed in the protein coding genes cox 1 and cob. The dynamic changes of these introns contributed to variations of mitochondrial genome size and organization. Introns were considered to be a mobile genetic element ${ }^{65}$, while homologous introns had the same insertion site in the coding region of PCGs ${ }^{43}$. The same Pcls had high sequence similarities, while different Pcls showed low similarities in nucleotide sequences. In the present study, the classes and number of Pcls were inconsistent in Polyporales. Some Pcls were widely distributed in Polyporales species, suggesting that these Pcls may be obtained from the common ancestor. However, some Pcls, e.g. Pcls Z and R, were observed only in one or two Polyporales species, which were also found in distant species such as A. bisporus and C. atmophyticus ${ }^{43}$, suggesting the possibility of horizontal gene transfer events. In general, the number and classes of introns were highly dynamic changes in the Polyporales species, which proved that numerous intron loss 
or gain events occurred in the evolution of Polyporales. The result was consistent with previous studies that the fungal group I introns appears to result from numerous losses and gains of the mobile genetic elements ${ }^{40,43,66,67}$.

The arrangement of mitochondrial genes can also serve as an important reference for revealing phylogenetic relationships among species ${ }^{68-70}$. In this study, we found that the arrangement of mitochondrial genes was highly variable in Polyporales. Gene orders between Ganoderma species were found highly conserved, while large-scale gene rearrangements were observed at family levels. Gene order analysis revealed that large-scale gene rearrangements occurred in T. camphoratus mitogenome compared with other Polyporales, involving protein-coding genes, tRNA genes, and rRNA genes, resulting in unique gene arrangement in T. camphoratus mitogenome. Previous studies have shown that the accumulation of repetitive sequences in the mitochondrial genome of fungi contributed to gene recombination of the fungal mitogenome, which in turn led to the rearrangement of mitochondrial genes $^{61}$. In this study, high proportion of repeats $(9.44 \%)$ were observed in the mitogenome of T. camphoratus. It is reasonably speculated that the accumulation of repetitive sequences in the mitogenome of T. camphoratus led to gene rearrangement in T. camphoratus. The effect of the repeat sequences accumulation in T. camphoratus mitogenome on the evolution and variation of T. camphoratus mitogenome needs to be further revealed.

Mitogenomes are thought to be derived from the common ancestral alpha-proteobacterium though endosymbiosis ${ }^{71,72}$. During evolution, most mitochondrial genes have been transferred into the nuclear genome, and this phenomenon was considered with multiple advantages ${ }^{73,74}$. However, fungal mitogenomes also retained a number of genes for energy metabolism and transcriptional regulation, including atp6, atp 8, atp $9, \operatorname{cob}, \operatorname{cox} 1, \cos 2$, cox3, nad1, nad2, nad3, nad4, nad4L, nad5, nad6, and rps3, which we call the core PCGs. We found that the core PCGs of Polyporales varied greatly in base composition and gene length. The effect of these variations on fungal adaptation to environment and energy metabolism remains unknown. In addition, a series of ORFs were found in the T. camphoratus mitogenome, which show low sequence similarities to known proteins in public database, suggesting that there are many unknown protein-coding genes in the T. camphoratus mitogenome that need to be revealed, which will facilitate understanding of the origin, evolution and function of fungal mitogenomes. Genetic distance analysis showed that nad4L gene was highly conserved among Polyporales species, while rps3 gene was highly variable in Polyporales. We found that most of the core PCGs in Polyporales were subjected to purifying selection. However, rps3 gene was under strong positive selection pressure between some species, which may be to better adapt to their lifestyles or surrounding environment ${ }^{49}$.

Because of the limited and confusing macroscopic and microscopic morphological characteristics of macrofungi, it is difficult to precise classify fungal species and subspecies, which limits the development and utilization of fungi. The introduction of molecular markers promotes the taxonomy of fungi. Mitochondrial genomes have been widely used in phylogenetics, evolutionary and population genetics because of rapid evolution rates and many available molecular markers ${ }^{75,76}$. Mitochondrial cox 1 gene and rRNA genes have been widely used in phylogenetic studies of animals $\mathrm{s}^{77,78}$. However, there are few reports on the application of mitochondrial genome in fungal taxonomy or phylogenetics. In the present study, we obtained a high-support phylogenetic tree based on the combined mitochondrial gene set, which divided the 25 Agaricomycetes species into four major clades that is consistent with previous studies ${ }^{1,20,63}$. T. camphoratus was found to be a sister species of L. sulphureus and closely related to F. palustris ${ }^{37}$. The results showed that mitochondrial genes were suitable as molecular markers for phylogenetic analysis of Agaricomycetes. More fungal mitochondrial genomes need to be revealed in the future to facilitate the study of fungal taxonomy, phylogenetics and population genetics.

\section{Materials and methods}

Assembly and annotation of $T$. camphoratus mitogenome. The whole genome sequencing reads of T. camphoratus used for mitogenome assembly were downloaded from NCBI SRA database under the accession number (SRR1258102) ${ }^{7}$. De novo assembly of the T. camphoratus mitogenome was performed as implemented by SPAdes $3.9^{79}$ with a kmer size of 17 , using the downloaded data. We used MITObim V1.9 ${ }^{80}$ to fill gaps among contigs. The obtained complete mitogenome of T. camphoratus was initially annotated combining the results of mfannot tool (https://megasun.bch.umontreal.ca/cgi-bin/dev_mfa/mfannotInterface.pl) ${ }^{81}$ and MITOS ${ }^{82}$, both based on the genetic code 4. At this step, protein coding genes (PCGs), tRNA genes and rRNA genes are preliminarily annotated. PCGs were also predicted or modified using the NCBI Open Reading Frame Finder (https ://www.ncbi.nlm.nih.gov/orffinder/) and annotated via BLASTP searches against the NCBI non-redundant protein sequence database ${ }^{83}$. PCGs which have no significant similarity to previously characterized proteins were annotated as hypothetical proteins. Intron-exon borders of PCGs were verified using exonerate v2.2 ${ }^{84}$. tRNAgenes were also predicted using the tRNAscan-SE 1.3.1 program $^{85}$.

Sequence analysis. DNASTAR Lasergene v7.1 (https://www.dnastar.com/) was used to analyze the base composition of the T. camphoratus mitogenome. Strand asymmetry of the mitogenome was assessed according to the following formulas: AT skew $=[A-T] /[A+T]$, and $G C$ skew $=[G-C] /[G+C]^{86}$. We used the DnaSP v6.10.01 $1^{87}$ software to calculate the synonymous substitution rate (Ks) and the nonsynonymous substitution rate (Ka) for all core PCGs in the T. camphoratus mitogenome, as well as in the previously published Polyporales mitogenomes.

The genetic distances between each pair of the 14 core PCGs (atp6, atp8, atp9, cox1, cox2, cox3, nad1, nad2, nad3, nad4, nad4L, nad5, nad6, and $c o b$ ) and the ribosomal protein S3 (rps3) gene were calculated with MEGA v6.06 ${ }^{88}$, using the Kimura-2-parameter (K2P) model. Codon usage were analyzed using the Sequence Manipulation Suite ${ }^{89}$, based on genetic code 4 . Genome synteny of the T. camphoratus mitogenome and its closely related species were analyzed with the Mauve v2.4.0 $0^{90}$. 
Identification of repetitive elements. BLASTn searches of the whole mitogenome against itself were performed to determine whether there was intra-genomic duplication of large fragments and interspersed repeats in the T. camphoratus mitogenome at an E value of $<10^{-10}$. In addition, tandem repeats $(>10$ bp in length) were detected using the online program Tandem Repeats Finder with default parameters ${ }^{91}$. Repeated sequences were also searched by REPuter to identify forward (direct), reverse, complemented, and palindromic (revere complemented) repeats ${ }^{92}$.

Comparative mitogenomic analysis and intron analysis. The genome sizes, GC content, base composition, and gene numbers were compared among different Polyporales species to assess variations and conservation among mitogenomes. Group I introns in cox 1 and cob genes of the 12 Polyporales species we detected were classified into different position classes (Pcls) according to the method described by Férandon et al. ${ }^{44}$. Each Pcl was constituted by introns inserted at the same position in the coding region of the cox 1 or the cob gene. The Pcls of coxl gene were named in letter according to the similarity with the described Pcls ${ }^{44}$. Pcls of $c o b$ gene were named in Latin number according to the insert position in the coding region of the cob gene.

Phylogenetic analysis. In order to investigate the phylogenetic status of T. camphoratus among Agaricomycetes class, we constructed a phylogenetic tree of 25 Agaricomycetes species based on the combined mitochondrial gene set (15 core PCGs +2 rRNA genes). Single mitochondrial gene was first aligned using MAFFT $\mathrm{v} 7.037^{93}$. And then we concatenated these alignments to gene set using SequenceMatrix v1.7.8 $8^{94}$. In order to detect potential phylogenetic conflicts between different genes, we carried out a preliminary partition homogeneity test. Best-fit models of evolution and partitioning schemes for the gene set were determined according to PartitionFinder 2.1.195. MrBayes v3.2.6 ${ }^{96}$ was used to construct the phylogenetic tree using Bayesian inference (BI) method based on the combined gene set. Two independent runs with four chains (three heated and one cold) each were conducted simultaneously for $2 \times 10^{6}$ generations. Each run was sampled every 100 generations. We assumed that stationarity had been reached when estimated sample size (ESS) was greater than 100, and the potential scale reduction factor (PSRF) approached 1.0. The first $25 \%$ samples were discarded as burn-in, and the remaining trees were used to calculate Bayesian posterior probabilities (BPP) in a $50 \%$ majority-rule consensus tree $^{37}$. We also constructed the phylogenetic tree using maximum likelihood (ML) method based on the combined gene set using RAxML v8.0.0 $0^{97}$.

Ethical approval. This article does not contain any studies with human participants performed by any of the authors.

\section{Data availability}

The T. camphoratus mitochondrial genome sequences were submitted to GenBank under accession number MH745717.

Received: 6 February 2020; Accepted: 8 September 2020

Published online: 05 October 2020

\section{References}

1. Wu, S. H. et al. Taiwanofungus, a polypore new genus. Fungal Sci. 19, 109-116 (2004).

2. Chen, L. Y. et al. Taiwanofungus camphoratus (Syn Antrodia camphorata) extract and amphotericin B exert adjuvant effects via mitochondrial apoptotic pathway. Integr. Cancer Ther. 12, 153-164 (2013).

3. Chen, L. Y. et al. Pretreatment with an ethanolic extract of Taiwanofungus camphoratus (Antrodia camphorata) enhances the cytotoxic effects of amphotericin B. J. Agric. Food Chem. 59, 11255-11263 (2011).

4. Chen, Y. F. et al. Zhankuic acid A isolated from Taiwanofungus camphoratus is a novel selective TLR4/MD-2 antagonist with antiinflammatory properties. J. Immunol. 192, 2778-2786 (2014).

5. Chen, Y. Y., Lo, C. P., Lin, C. C. \& Hsieh, Y. H. Effects of Taiwanofungus camphoratus on non-specific and specific immune activities in mice. Mycology 9, 129-135 (2018).

6. Hsieh, Y. H. et al. Antrocamphin A, an anti-inflammatory principal from the fruiting body of Taiwanofungus camphoratus, and its mechanisms. J. Agric. Food Chem. 58, 3153-3158 (2010).

7. Lu, M. Y. et al. Genomic and transcriptomic analyses of the medicinal fungus Antrodia cinnamomea for its metabolite biosynthesis and sexual development. Proc. Natl. Acad. Sci. USA 111, E4743-E4752 (2014).

8. Shi, L. S. et al. Biologically active constituents from the fruiting body of Taiwanofungus camphoratus. Bioorg. Med. Chem. 19, 677-683 (2011).

9. Liu, D. Z. et al. Comparative anti-inflammatory characterization of wild fruiting body, liquid-state fermentation, and solid-state culture of Taiwanofungus camphoratus in microglia and the mechanism of its action. J. Ethnopharmacol. 113, 45-53 (2007).

10. Lin, L. T. et al. The Ethanolic extract of Taiwanofungus camphoratus (Antrodia camphorata) induces cell cycle arrest and enhances cytotoxicity of cisplatin and doxorubicin on human hepatocellular carcinoma cells. Biomed. Res. Int. 2015, 415269 (2015).

11. Lee, K. H. et al. Recent progress of research on medicinal mushrooms, foods, and other herbal products used in traditional Chinese medicine. J. Tradit. Complement. Med. 2, 84-95 (2012).

12. Hatefi, Y. ATP synthesis in mitochondria. Eur. J. Biochem. 218, 759-767 (1993).

13. Gray, M. W., Burger, G. \& Lang, B. F. Mitochondrial evolution. Science 283, 1476-1481 (1999).

14. Saccone, C. The evolution of mitochondrial DNA. Curr. Opin. Genet. Dev. 4, 875-881 (1994).

15. Chen, X. J. \& Butow, R. A. The organization and inheritance of the mitochondrial genome. Nat. Rev. Genet. 6, 815-825 (2005).

16. Nadimi, M., Daubois, L. \& Hijri, M. Mitochondrial comparative genomics and phylogenetic signal assessment of mtDNA among arbuscular mycorrhizal fungi. Mol. Phylogenet. Evol. 98, 74-83 (2016).

17. Burger, T. D., Shao, R., Labruna, M. B. \& Barker, S. C. Molecular phylogeny of soft ticks (Ixodida: Argasidae) inferred from mitochondrial genome and nuclear rRNA sequences. Ticks Tick Borne Dis. 5, 195-207 (2014).

18. Li, Q. et al. Characterization and comparative mitogenomic analysis of six newly sequenced mitochondrial genomes from ectomycorrhizal fungi (Russula) and phylogenetic analysis of the Agaricomycetes. Int. J. Biol. Macromol. 119, 792-802 (2018). 
19. Li, Q., Wang, Q., Jin, X., Chen, Z., Xiong, C. \& Li, P. et al. The first complete mitochondrial genome from the family Hygrophoraceae (Hygrophorus russula) by next-generation sequencing and phylogenetic implications. Int. J. Biol. Macromol. 122, 1313-1320 (2019).

20. Li, Q. et al. Characterization and comparison of the mitochondrial genomes from two Lyophyllum fungal species and insights into phylogeny of Agaricomycetes. Int. J. Biol. Macromol. 121, 364-372 (2018).

21. Li, Q. et al. Comparative mitogenome analysis of two ectomycorrhizal fungi (Paxillus) reveals gene rearrangement, intron dynamics, and phylogeny of basidiomycetes. IMA Fungus 11, 12 (2020).

22. Li, Q. et al. Comparative mitogenome analysis reveals mitochondrial genome differentiation in ectomycorrhizal and asymbiotic Amanita species. Front. Microbiol. 11, 1382 (2020).

23. Du, Y., Dietrich, C.H. \& Dai, W. Complete mitochondrial genome of Macrosteles quadrimaculatus (Matsumura) (Hemiptera: Cicadellidae: Deltocephalinae) with a shared tRNA rearrangement and its phylogenetic implications. Int. J. Biol. Macromol. 122, 1027-1034 (2019).

24. Huang, Y., Liu, Y., Zhu, X. Y., Xin, Z. Z., Zhang, H. B. \& Zhang, D. Z. et al. Comparative mitochondrial genome analysis of Grammodes geometrica and other noctuid insects reveals conserved mitochondrial genome organization and phylogeny. Int. J. Biol. Macromol. 125, 1257-1265 (2019).

25. Su, T. \& Liang, A. Characterization of the complete mitochondrial genome of Phymatostetha huangshanensis (Hemiptera: Cercopidae) and phylogenetic analysis. Int. J. Biol. Macromol. 119, 60-69 (2018).

26. Bronstein, O. \& Kroh, A. The first mitochondrial genome of the model echinoid Lytechinus variegatus and insights into Odontophoran phylogenetics. Genomics 111, 710-718 (2019).

27. Hawksworth, D. L. \& Lucking, R. Fungal diversity revisited: 2.2 to 3.8 million species. Microbiol. Spectr. 5, FUNK-0052-2016 (2017).

28. Lindahl, B. D. et al. Spatial separation of litter decomposition and mycorrhizal nitrogen uptake in a boreal forest. New Phytol. 173, 611-620 (2007).

29. Barea, J. M., Pozo, M. J., Azcon, R. \& Azcon-Aguilar, C. Microbial co-operation in the rhizosphere. J. Exp. Bot. 56, 1761-1778 (2005).

30. Saarma, U. et al. A novel phylogeny for the genus Echinococcus, based on nuclear data, challenges relationships based on mitochondrial evidence. Parasitology 136, 317-328 (2009).

31. Li, J. et al. Complete mitochondrial genome of the medicinal mushroom Ganoderma lucidum. PLoS ONE 8, e72038 (2013).

32. Wang, X. C., Shao, J. \& Liu, C. The complete mitochondrial genome of the medicinal fungus Ganoderma applanatum (Polyporales, Basidiomycota). Mitochondrial DNA A DNA Mapp. Seq. Anal. 27, 2813-2814 (2016).

33. Wang, X. C. et al. The complete mitochondrial genome of the white-rot fungus Ganoderma meredithiae (Polyporales, Basidiomycota). Mitochondrial DNA A DNA Mapp. Seq. Anal. 27, 4197-4198 (2016).

34. Li, Q. et al. The complete mitochondrial genomes of five important medicinal Ganoderma species: Features, evolution, and phylogeny. Int. J. Biol. Macromol. 139, 397-408 (2019).

35. Haridas, S. \& Gantt, J. S. The mitochondrial genome of the wood-degrading basidiomycete Trametes cingulata. FEMS Microbiol. Lett. 308, 29-34 (2010).

36. Salavirta, H. et al. Mitochondrial genome of Phlebia radiata is the second largest (156 kbp) among fungi and features signs of genome flexibility and recent recombination events. PLoS ONE 9, e97141 (2014).

37. $\mathrm{Li}$, Q. et al. Characterization and phylogenetic analysis of the complete mitochondrial genome of the medicinal fungus Laetiporus sulphureus. Sci. Rep. 8, 9104 (2018).

38. Tanaka, Y. et al. The complete genome sequence and phylogenetic analysis of the mitochondrial DNA of the wood-decaying fungus Fomitopsis palustris. Genes Genomics 39, 1377-1385 (2017).

39. Li, Q. et al. Comparative mitogenomics reveals large-scale gene rearrangements in the mitochondrial genome of two Pleurotus species. Appl. Microbiol. Biotechnol. 102, 6143-6153 (2018).

40. Gomes, F. et al. Polymorphism in mitochondrial group I introns among Cryptococcus neoformans and Cryptococcus gattii genotypes and its association with drug susceptibility. Front. Microbiol. 9, 86 (2018).

41. Novikova, O. \& Belfort, M. Mobile group II introns as ancestral eukaryotic elements. Trends Genet. 33, 773-783 (2017).

42. Li, Q. et al. The complete mitochondrial genomes of two model ectomycorrhizal fungi (Laccaria): Features, intron dynamics and phylogenetic implications. Int. J. Biol. Macromol. 145, 974-984 (2020).

43. Ferandon, C., Xu, J. \& Barroso, G. The $135 \mathrm{kbp}$ mitochondrial genome of Agaricus bisporus is the largest known eukaryotic reservoir of group I introns and plasmid-related sequences. Fungal Genet. Biol. 55, 85-91 (2013).

44. Ferandon, C. et al. The Agaricus bisporus coxl gene: The longest mitochondrial gene and the largest reservoir of mitochondrial group I introns. PLoS ONE 5, e14048 (2010).

45. Li, Q. et al. Comparative mitochondrial genome analysis of two ectomycorrhizal fungi (Rhizopogon) reveals dynamic changes of intron and phylogenetic relationships of the subphylum agaricomycotina. Int. J. Mol. Sci. 20, 5167 (2019).

46. Wang, X. et al. The $206 \mathrm{kbp}$ mitochondrial genome of Phanerochaete carnosa reveals dynamics of introns, accumulation of repeat sequences and plasmid-derived genes. Int. J. Biol. Macromol. 162, 209-219 (2020).

47. Li, Q. et al. Characterization and comparative analysis of six complete mitochondrial genomes from ectomycorrhizal fungi of the Lactarius genus and phylogenetic analysis of the Agaricomycetes. Int. J. Biol. Macromol. 121, 249-260 (2018).

48. Fourie, G. et al. Mitochondrial introgression and interspecies recombination in the Fusarium fujikuroi species complex. IMA Fungus 9, 37-48 (2018).

49. Korovesi, A. G., Ntertilis, M. \& Kouvelis, V. N. Mt-rps3 is an ancient gene which provides insight into the evolution of fungal mitochondrial genomes. Mol. Phylogenet. Evol. 127, 74-86 (2018).

50. Costa, G. G. et al. The mitochondrial genome of Moniliophthora roreri, the frosty pod rot pathogen of cacao. Fungal Biol. 116, 551-562 (2012).

51. Formighieri, E. F. et al. The mitochondrial genome of the phytopathogenic basidiomycete Moniliophthora perniciosa is $109 \mathrm{~kb}$ in size and contains a stable integrated plasmid. Mycol. Res. 112, 1136-1152 (2008).

52. Barroso, G., Blesa, S. \& Labarere, J. Wide distribution of mitochondrial genome rearrangements in wild strains of the cultivated basidiomycete Agrocybe aegerita. Appl. Environ. Microbiol. 61, 1187-1193 (1995).

53. Ye, J., Cheng, J., Ren, Y., Liao, W. \& Li, Q. The first mitochondrial genome for geastrales (Sphaerobolus stellatus) reveals intron dynamics and large-scale gene rearrangements of Basidiomycota. Front. Microbiol. 11, 1970 (2020).

54. Alexeyev, M. F., Ledoux, S. P. \& Wilson, G. L. Mitochondrial DNA and aging. Clin. Sci. (Lond.) 107, 355-364 (2004).

55. Burgstaller, J. P., Johnston, I. G. \& Poulton, J. Mitochondrial DNA disease and developmental implications for reproductive strategies. Mol. Hum. Reprod. 21, 11-22 (2015).

56. Taylor, R. W. \& Turnbull, D. M. Mitochondrial DNA mutations in human disease. Nat. Rev. Genet. 6, 389-402 (2005).

57. Sulo, P. et al. The evolutionary history of Saccharomyces species inferred from completed mitochondrial genomes and revision in the "yeast mitochondrial genetic code". DNA Res. 24, 571-583 (2017).

58. van Esveld, S. L. \& Huynen, M. A. Does mitochondrial DNA evolution in metazoa drive the origin of new mitochondrial proteins? IUBMB Life 70, 1240-1250 (2018).

59. Turanov, S. V., Lee, Y. H. \& Kartavtsev, Y. P. Structure, evolution and phylogenetic informativeness of eelpouts (Cottoidei: Zoarcales) mitochondrial control region sequences. Mitochondrial DNA A DNA Mapp. Seq. Anal. 30, 264-272 (2019).

60. Smith, D. R. The past, present and future of mitochondrial genomics: Have we sequenced enough mtDNAs?. Brief. Funct. Genomics 15, 47-54 (2016). 
61. Aguileta, G. et al. High variability of mitochondrial gene order among fungi. Genome Biol. Evol. 6, 451-465 (2014).

62. Ward, B. L., Anderson, R. S. \& Bendich, A. J. The mitochondrial genome is large and variable in a family of plants (cucurbitaceae). Cell 25, 793-803 (1981).

63. Li, Q. et al. Characterization of the mitochondrial genomes of three species in the ectomycorrhizal genus Cantharellus and phylogeny of Agaricomycetes. Int. J. Biol. Macromol. 118, 756-769 (2018).

64. Losada, L. et al. Mobile elements and mitochondrial genome expansion in the soil fungus and potato pathogen Rhizoctonia solani AG-3. FEMS Microbiol. Lett. 352, 165-173 (2014).

65. Fan, W. W., Zhang, S. \& Zhang, Y. J. The complete mitochondrial genome of the Chan-hua fungus Isaria cicadae: A tale of intron evolution in Cordycipitaceae. Environ. Microbiol. 21, 864-879 (2019).

66. Deng, Y. et al. Comparison of the mitochondrial genome sequences of six Annulohypoxylon stygium isolates suggests short fragment insertions as a potential factor leading to larger genomic size. Front. Microbiol. 9, 2079 (2018).

67. Wang, L., Zhang, S., Li, J. H. \& Zhang, Y. J. Mitochondrial genome, comparative analysis and evolutionary insights into the entomopathogenic fungus Hirsutella thompsonii. Environ. Microbiol. 20, 3393-3405 (2018).

68. Desalle, R., Schierwater, B. \& Hadrys, H. MtDNA: The small workhorse of evolutionary studies. Front. Biosci. (Landmark Ed) 22, 873-887 (2017).

69. Delsuc, F., Stanhope, M. J. \& Douzery, E. J. Molecular systematics of armadillos (Xenarthra, Dasypodidae): Contribution of maximum likelihood and Bayesian analyses of mitochondrial and nuclear genes. Mol. Phylogenet. Evol. 28, 261-275 (2003).

70. Zhong, L. et al. Complete mitochondrial genome of Odontobutis haifengensis (Perciformes, Odontobutiae): A unique rearrangement of tRNAs and additional non-coding regions identified in the genus Odontobutis. Genomics 110, 382-388 (2018).

71. Zachar, I. \& Szathmary, E. Breath-giving cooperation: Critical review of origin of mitochondria hypotheses: Major unanswered questions point to the importance of early ecology. Biol. Direct. 12, 19 (2017).

72. Dunn, C. D. Some liked it hot: a hypothesis regarding establishment of the proto-mitochondrial endosymbiont during Eukaryogenesis. J. Mol. Evol. 85, 99-106 (2017).

73. Adams, K. L. \& Palmer, J. D. Evolution of mitochondrial gene content: Gene loss and transfer to the nucleus. Mol. Phylogenet. Evol. 29, 380-395 (2003).

74. Adams, K. L., Qiu, Y. L., Stoutemyer, M. \& Palmer, J. D. Punctuated evolution of mitochondrial gene content: High and variable rates of mitochondrial gene loss and transfer to the nucleus during angiosperm evolution. Proc. Natl. Acad. Sci. USA 99, 9905-9912 (2002).

75. Doyle, J. M. et al. New insights into the phylogenetics and population structure of the prairie falcon (Falco mexicanus). BMC Genomics 19, 233 (2018)

76. Dai, L. S., Kausar, S., Abbas, M. N. \& Wang, T. T. Complete sequence and characterization of the Ectropis oblique mitochondrial genome and its phylogenetic implications. Int. J. Biol. Macromol. 107, 1142-1150 (2018).

77. Yuan, M. L. et al. Mitochondrial phylogeny, divergence history and high-altitude adaptation of grassland caterpillars (Lepidoptera: Lymantriinae: Gynaephora) inhabiting the Tibetan Plateau. Mol. Phylogenet. Evol. 122, 116-124 (2018).

78. Ananthan, G. \& Murugan, R. Molecular phylogeny of four ascidian species inferred from mitochondrial Cytochrome Oxidase subunit I (COI) sequence. Mitochondrial DNA A DNA Mapp. Seq. Anal. 29, 121-125 (2018).

79. Bankevich, A. et al. SPAdes: A new genome assembly algorithm and its applications to single-cell sequencing. J. Comput. Biol. 19, $455-477$ (2012).

80. Hahn, C., Bachmann, L. \& Chevreux, B. Reconstructing mitochondrial genomes directly from genomic next-generation sequencing reads-A baiting and iterative mapping approach. Nucleic Acids Res. 41, e129 (2013).

81. Valach, M., Burger, G., Gray, M. W. \& Lang, B. F. Widespread occurrence of organelle genome-encoded 5S rRNAs including permuted molecules. Nucleic Acids Res. 42, 13764-13777 (2014).

82. Bernt, M. et al. MITOS: Improved de novo metazoan mitochondrial genome annotation. Mol. Phylogenet. Evol. 69, 313-319 (2013).

83. Bleasby, A. J. \& Wootton, J. C. Construction of validated, non-redundant composite protein sequence databases. Protein Eng. 3, $153-159$ (1990).

84. Slater, G. S. \& Birney, E. Automated generation of heuristics for biological sequence comparison. BMC Bioinform. 6, 31 (2005).

85. Lowe, T. M. \& Chan, P. P. tRNAscan-SE On-line: Integrating search and context for analysis of transfer RNA genes. Nucleic Acids Res. 44, W54-W57 (2016).

86. Wang, J. et al. Comparative mitogenomic analysis of mirid bugs (Hemiptera: Miridae) and evaluation of potential DNA barcoding markers. PeerJ 5, e3661 (2017).

87. Rozas, J. et al. DnaSP 6: DNA sequence polymorphism analysis of large data sets. Mol. Biol. Evol. 34, 3299-3302 (2017).

88. Caspermeyer, J. MEGA evolutionary software re-engineered to handle today's big data demands. Mol. Biol. Evol. 33, 1887 (2016).

89. Stothard, $\mathrm{P}$. The sequence manipulation suite: JavaScript programs for analyzing and formatting protein and DNA sequences. Biotechniques 28, 1102-1104 (2000).

90. Darling, A. C., Mau, B., Blattner, F. R. \& Perna, N. T. Mauve: multiple alignment of conserved genomic sequence with rearrangements. Genome Res 14, 1394-1403 (2004).

91. Benson, G. Tandem repeats finder: a program to analyze DNA sequences. Nucleic Acids Res. 27, 573-580 (1999).

92. Kurtz, S. et al. REPuter: The manifold applications of repeat analysis on a genomic scale. Nucleic Acids Res. 29, 4633-4642 (2001).

93. Katoh, K., Rozewicki, J. \& Yamada, K. D. MAFFT online service: multiple sequence alignment, interactive sequence choice and visualization. Brief Bioinform. 20, 1160-1166 (2019).

94. Vaidya, G., Lohman, D. L. \& Meier, R. SequenceMatrix: Concatenation software for the fast assembly of multi-gene datasets with character set and codon information. Cladistics 27, 171-180 (2011).

95. Lanfear, R., Frandsen, P. B., Wright, A. M., Senfeld, T. \& Calcott, B. PartitionFinder 2: New methods for selecting partitioned models of evolution for molecular and morphological phylogenetic analyses. Mol. Biol. Evol. 34, 772-773 (2017).

96. Ronquist, F. et al. MrBayes 3.2: Efficient Bayesian phylogenetic inference and model choice across a large model space. Syst. Biol. 61, 539-542 (2012).

97. Stamatakis, A. RAxML version 8: A tool for phylogenetic analysis and post-analysis of large phylogenies. Bioinformatics 30, 1312-1313 (2014).

\section{Author contributions}

Conceived and designed experiments: Q.L., X.W., and N.L. Performed the experiments: L.J., M.W., and H.Y. Analyzed the data: Q.L., M.C., X.L., and H.L. Wrote and revised the paper: Q. L., and X. W.

\section{Funding}

This work was supported by Project of Henan Educational Department of China (18A180015, 18A210017), SciTech Project of Henan Province, China (182102110278), and Sci-Tech Innovation Fund of Henan Agricultural University (KJCX2019A16). And Open Project of State Key Laboratory of Crop Stress Biology for Arid Areas, NWAFU of China (CSBAAKF2018007). 


\section{Competing interests}

The authors declare no competing interests.

\section{Additional information}

Supplementary information is available for this paper at https://doi.org/10.1038/s41598-020-73461-x.

Correspondence and requests for materials should be addressed to Q.L. or N.L.

Reprints and permissions information is available at www.nature.com/reprints.

Publisher's note Springer Nature remains neutral with regard to jurisdictional claims in published maps and institutional affiliations.

Open Access This article is licensed under a Creative Commons Attribution 4.0 International License, which permits use, sharing, adaptation, distribution and reproduction in any medium or format, as long as you give appropriate credit to the original author(s) and the source, provide a link to the Creative Commons licence, and indicate if changes were made. The images or other third party material in this article are included in the article's Creative Commons licence, unless indicated otherwise in a credit line to the material. If material is not included in the article's Creative Commons licence and your intended use is not permitted by statutory regulation or exceeds the permitted use, you will need to obtain permission directly from the copyright holder. To view a copy of this licence, visit http://creativecommons.org/licenses/by/4.0/.

(c) The Author(s) 2020 